Supporting Information

\title{
Variations in electrochemical characteristics of platinum catalyst enwrapped by carbon shell according to carbon layer thickness
}

Dohyeon Lee ${ }^{1}$, Youngkwang Kim ${ }^{1,2}$, Jihyeok Song ${ }^{1}$, Hee Ji Choi ${ }^{3}$, Mohanraju Karuppannan ${ }^{1}$, Yong-Hun $\mathrm{Cho}^{3, *}$, and Oh Joong Kwon ${ }^{1, *}$

${ }^{1}$ Department of Energy and Chemical Engineering, Incheon National University, 119 Academyro, Yeonsu-gu, Incheon 22012, Republic of Korea

${ }^{2}$ School of Chemical and Biological Engineering, Seoul National University, 1 Gwanak-ro, Seoul 08826, Republic of Korea

${ }^{3}$ Department of Chemical Engineering, Kangwon National University, Samcheok 25913, Republic of Korea

*Corresponding authors

Email: yhun00@kangwon.ac.kr (Yong-Hun Cho), ojkwon@inu.ac.kr (Oh Joong Kwon)

KEYWORDS : carbon shell, oxidation, thickness control, fuel cell, activity, stability 

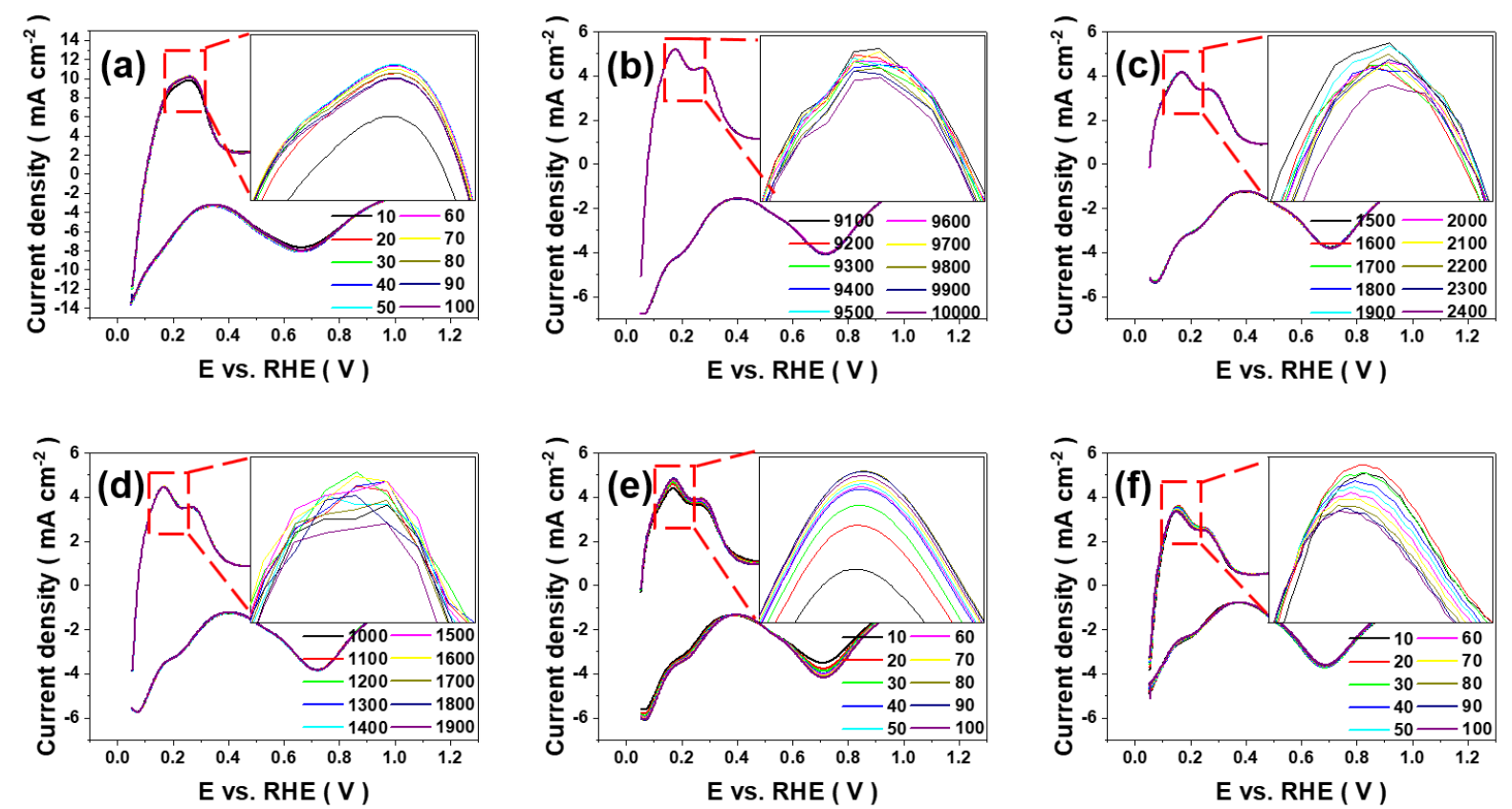

Figure S1. Activation cycles for reaching maximum current density measured by cyclic voltammetry in half cell with (a) Pt/C, (b) Pt@CS/CNF, (c) Pt@CS/CNF_5, (d) Pt@CS/CNF_10, (e) Pt@CS/CNF_20, and (f) Pt/CNF without carbon shell. 

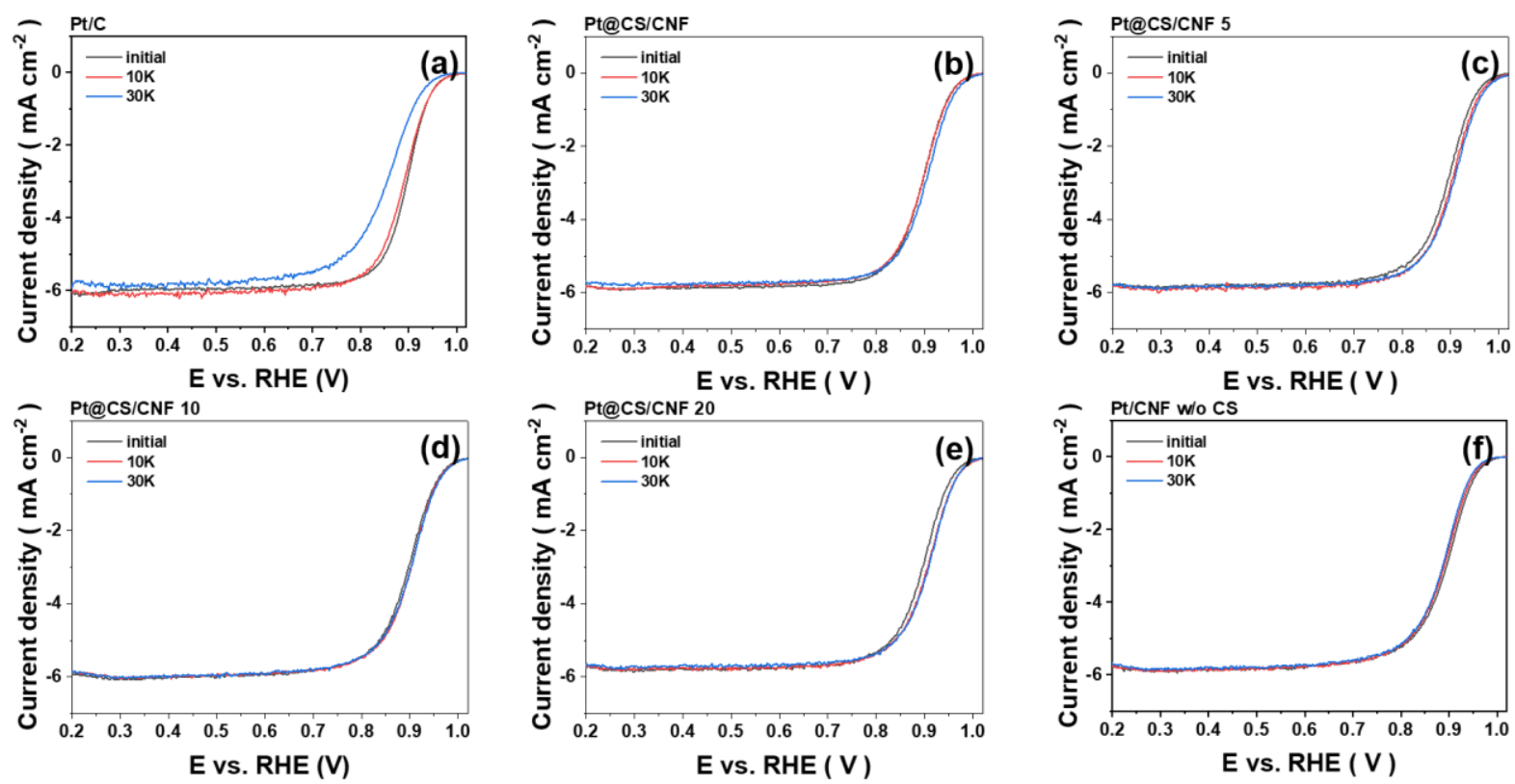

Figure S2. LSV curves for ORR obtained at different ADT cycles of 0 cycle, 10,000 cycles, and 30,000 cycles with different catalysts of (a) Pt/C, (b) Pt@CS/CNF, (c) Pt@CS/CNF_5, (d) Pt@CS/CNF_10, (e) Pt@CS/CNF_20, and (f) Pt/CNF without carbon shell. 


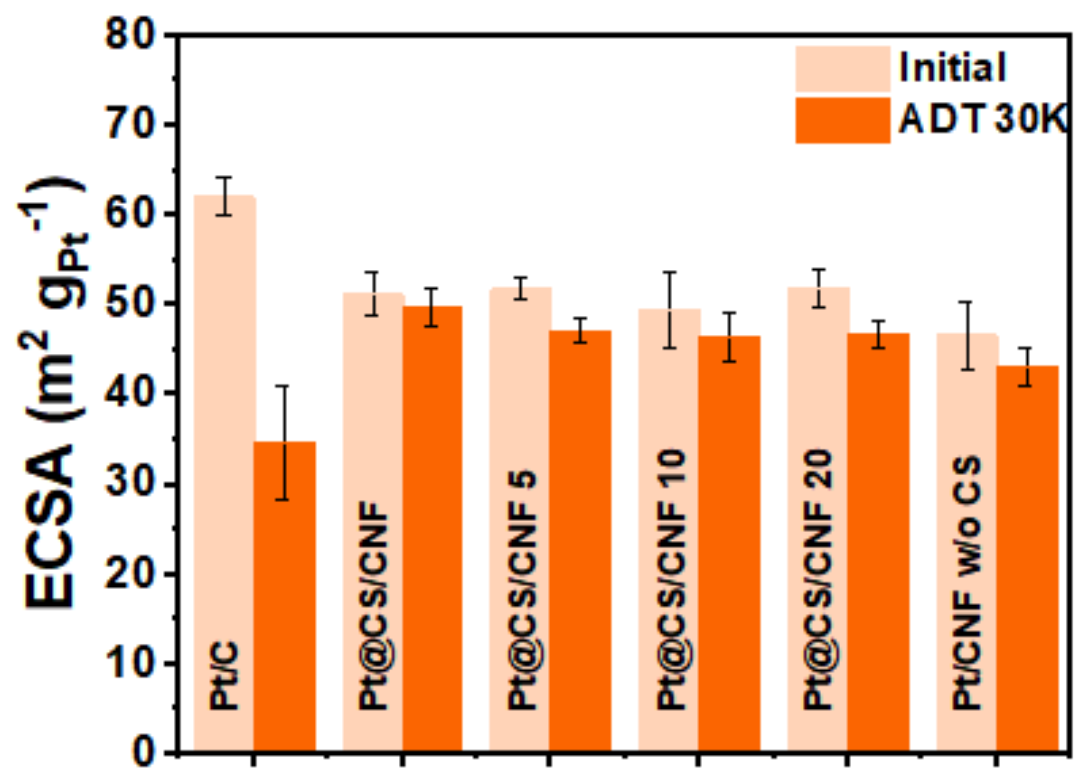

Figure S3. ECSAs of the catalysts before and after ADT in half cell tests 


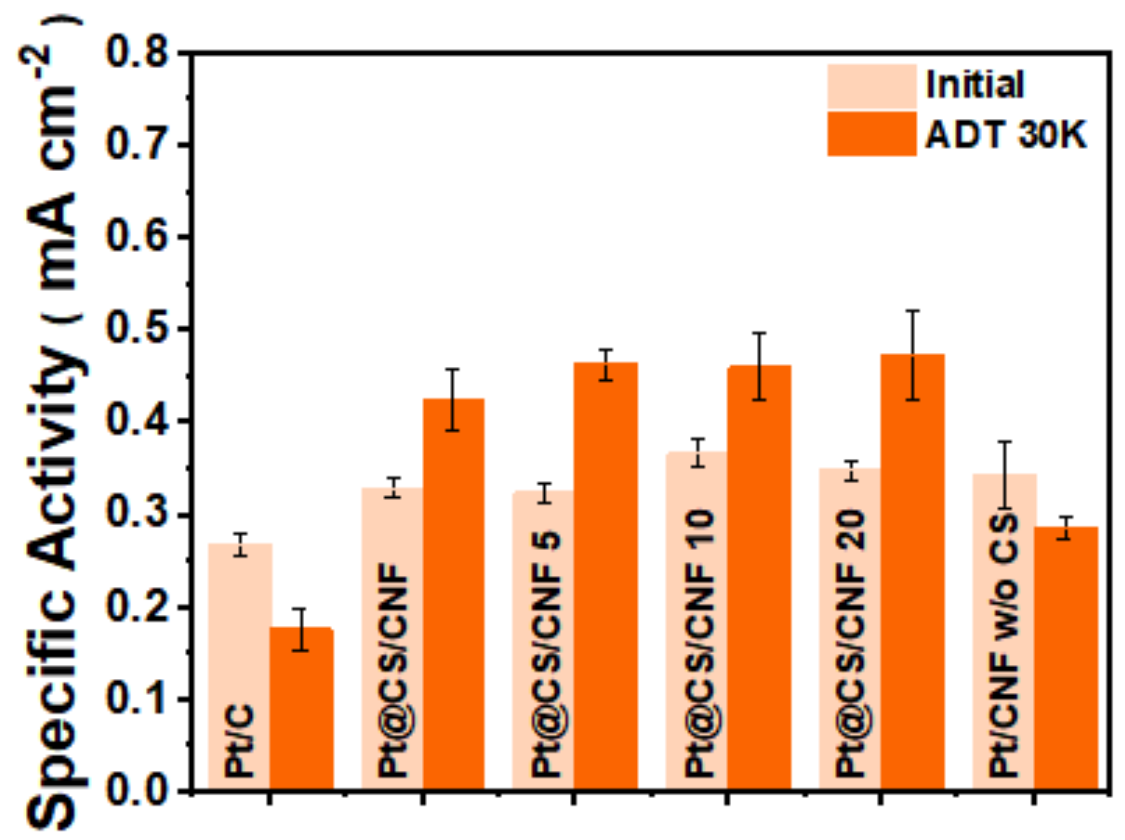

Figure S4. Specific Activity of the catalysts before and after ADT in half cell tests 


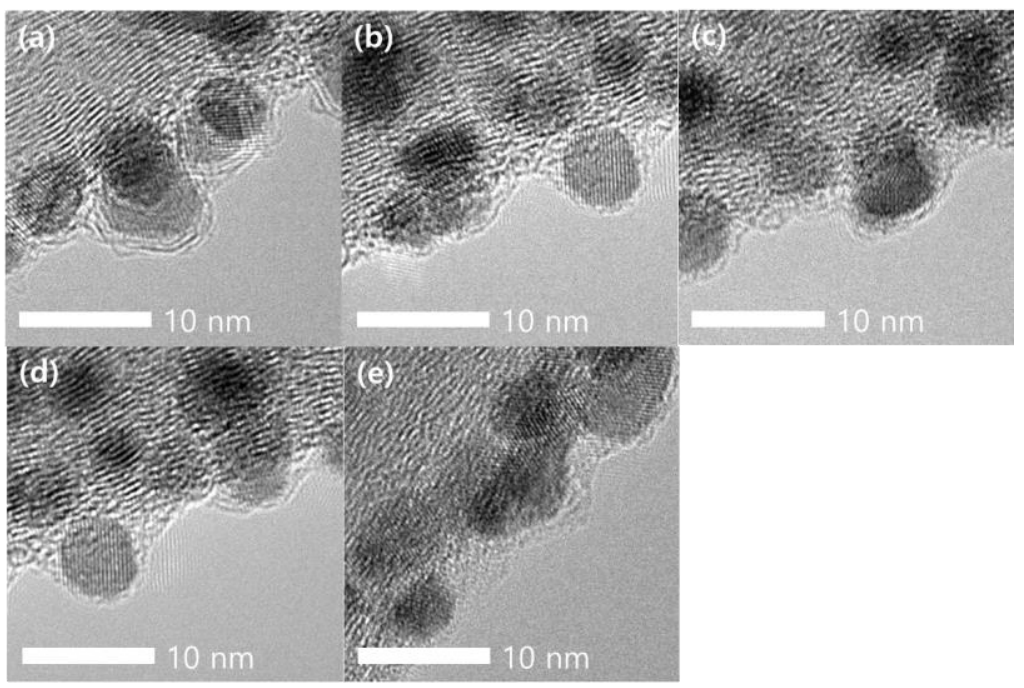

Figure S5. HR-TEM images of (a) Pt@CS/CNF, (b) Pt@CS/CNF_5, (c) Pt@CS/CNF_10, (d) $\mathrm{Pt} @ \mathrm{CS} / \mathrm{CNF} \_20$, and (e) Pt/CNF without carbon shell after optimized activation 


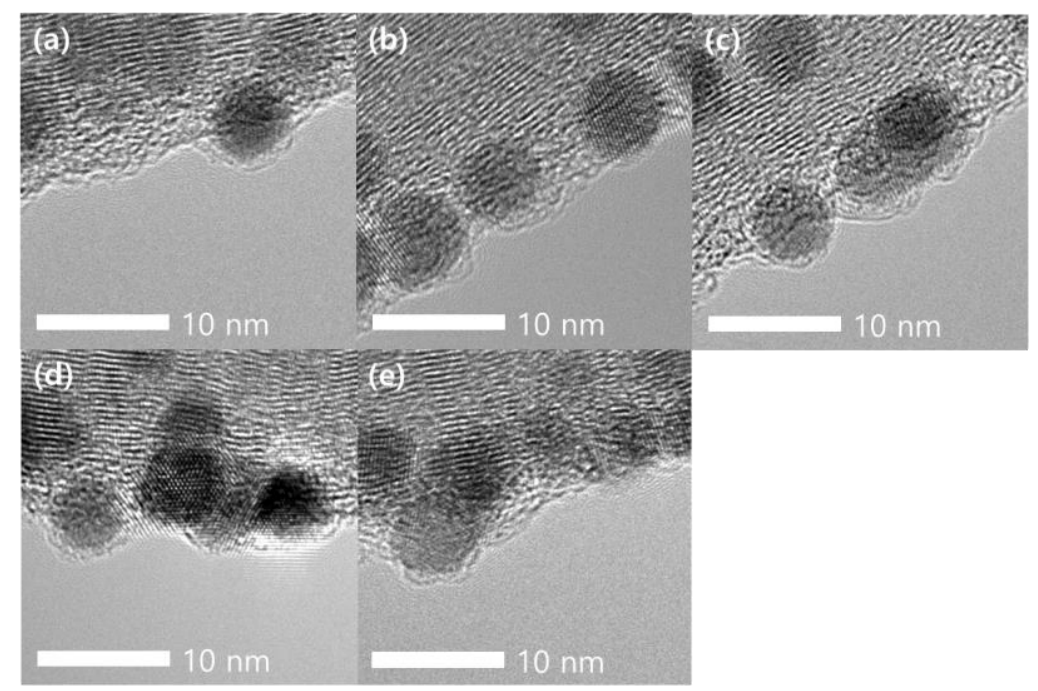

Figure S6. HR-TEM images of (a) Pt@CS/CNF, (b) Pt@CS/CNF_5, (c) Pt@CS/CNF_10, (d) Pt@CS/CNF_20, and (e) Pt/CNF without carbon shell at after 30K ADT in half cell 

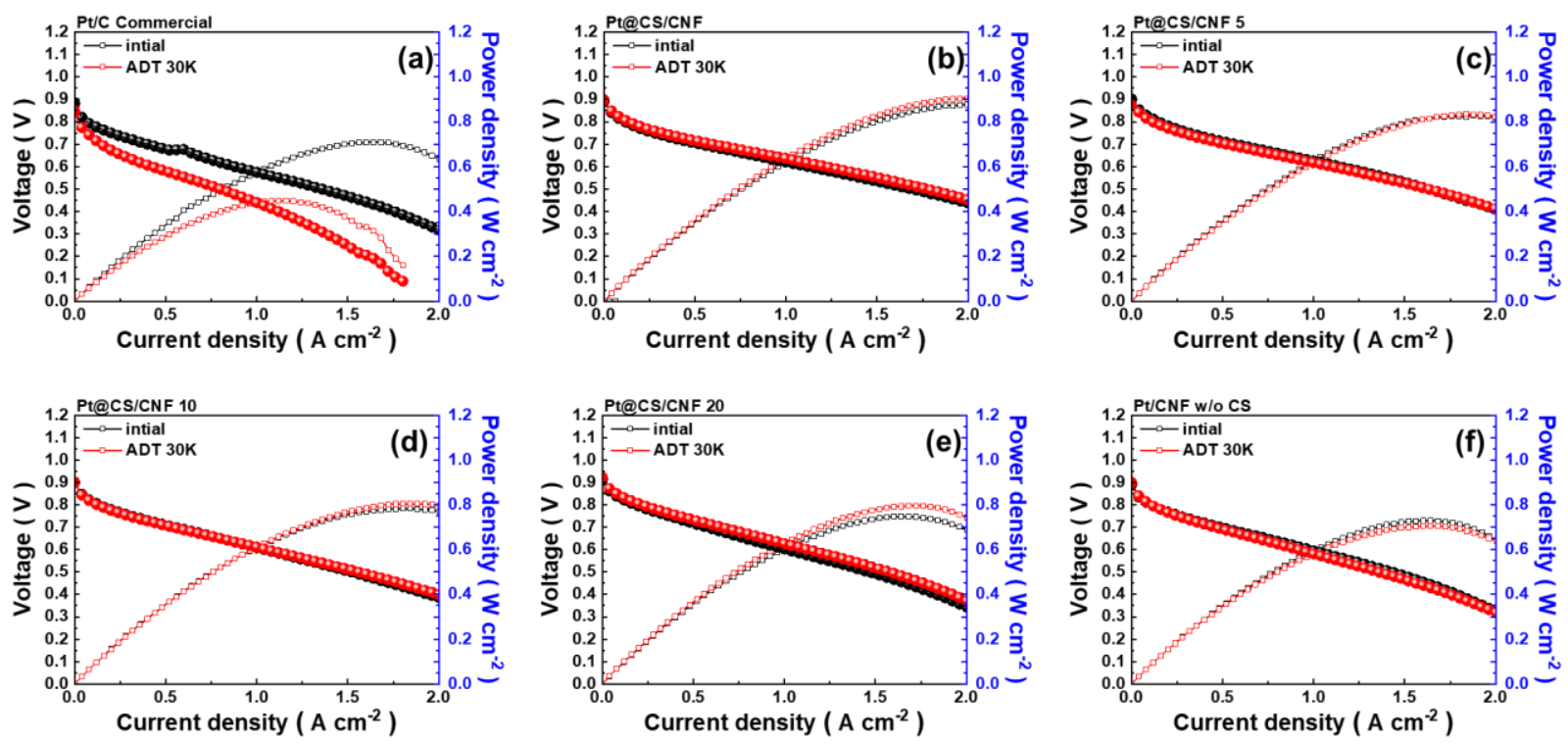

Figure S7. I-V curves of unit cells recorded at different ADT cycles of 0 cycle and 30,000 cycles with different catalysts of (a) Pt/C, (b) Pt@CS/CNF, (c) Pt@CS/CNF_5, (d) Pt@CS/CNF_10, (e) Pt@CS/CNF_20, and (f) Pt/CNF without carbon shell. 


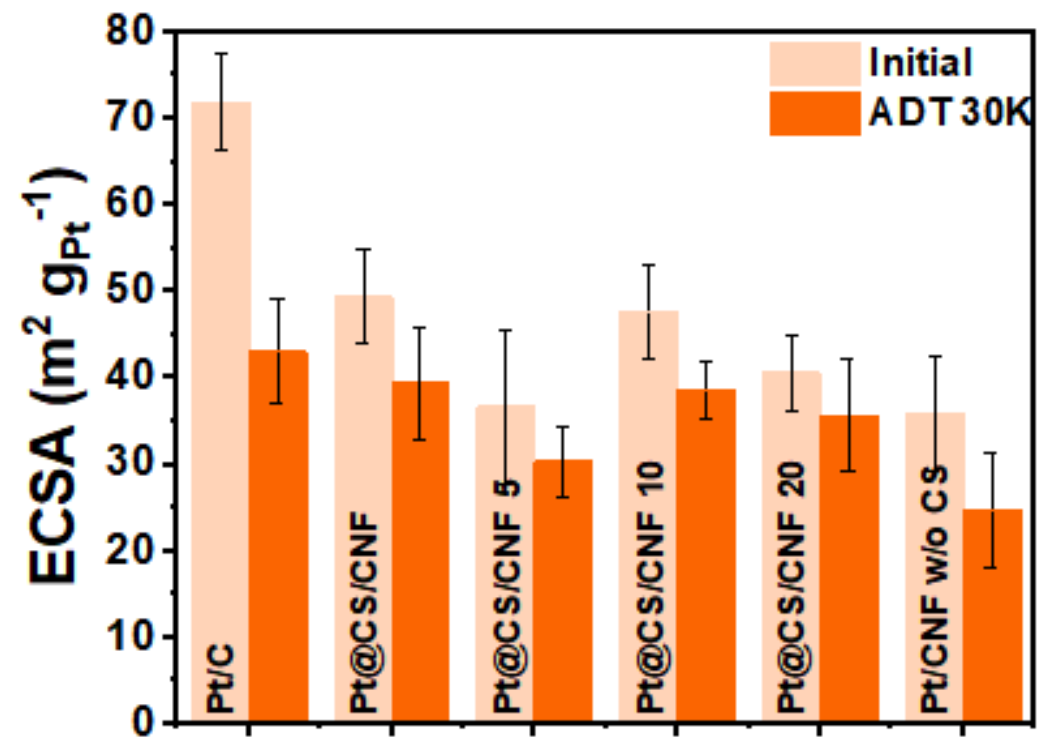

Figure S8. ECSAs of the catalysts before and after ADT in unit cell tests 


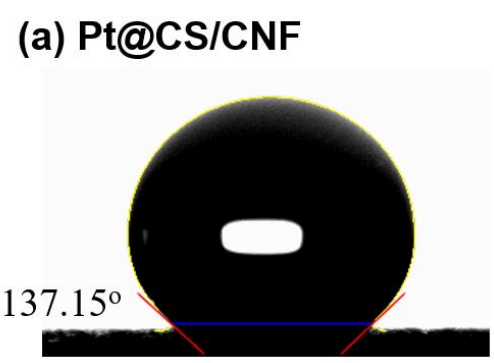

(d) Pt@CS/CNF 20 (b) Pt@CS/CNF 5

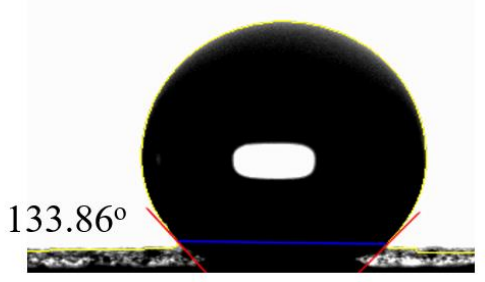

(e) Pt/CNF w/o CS (c) Pt@CS/CNF 10

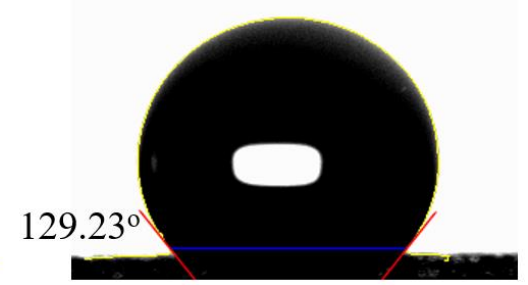

$117.59^{\circ}$

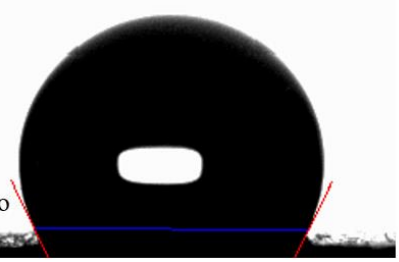

$127.87^{\circ}$

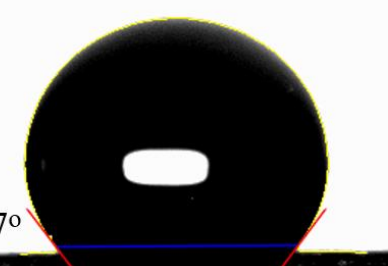

Figure S9. Contact angles observed on the different catalyst layers of (a) Pt/C, (b) Pt@CS/CNF,

(c)Pt@CS/CNF 5, (d) Pt@CS/CNF 10, (e) Pt@CS/CNF 20, and (f) Pt/CNF without carbon shell. 